\title{
In-depth investigations of adolescents and adults with holoprosencephaly identify unique characteristics
}

\author{
Karin Weiss, MD ${ }^{1}$, Paul Kruszka, MD1, Maria J. Guillen Sacoto, MD ${ }^{1}$, Yonit A. Addissie, BA ${ }^{1}$, \\ Donald W. Hadley, $\mathrm{MS}^{1}$, Casey K. Hadsall, $\mathrm{MS}^{1}$, Bethany Stokes, MS ${ }^{1}$, Ping $\mathrm{Hu}, \mathrm{ScD}^{1}$, \\ Erich Roessler, MD, PhD ${ }^{1}$, Beth Solomon, $\mathrm{MS}^{2}$, Edythe Wiggs, $\mathrm{PhD}^{3}$, Audrey Thurm, $\mathrm{PhD}^{4}$, \\ Robert B. Hufnagel, MD, PhD ${ }^{5}$, Wadih M. Zein, MD ${ }^{5}$, Jin S. Hahn, $\mathrm{MD}^{6}$, Elaine Stashinko, PhD \\ Eric Levey, $\mathrm{MD}^{7}$, Debbie Baldwin, $\mathrm{BS}^{8}$, Nancy J. Clegg, $\mathrm{PhD}^{8}$, Mauricio R. Delgado, $\mathrm{MD}^{8,9}$ and \\ Maximilian Muenke, $\mathrm{MD}^{1}$
}

\begin{abstract}
Purpose: With improved medical care, some individuals with holoprosencephaly (HPE) are surviving into adulthood. We investigated the clinical manifestations of adolescents and adults with HPE and explored the underlying molecular causes.

Methods: Participants included 20 subjects 15 years of age and older. Clinical assessments included dysmorphology exams, cognitive testing, swallowing studies, ophthalmic examination, and brain magnetic resonance imaging. Genetic testing included chromosomal microarray, Sanger sequencing for SHH, ZIC2, SIX3, and TGIF, and whole-exome sequencing (WES) of 10 trios.
\end{abstract}

Results: Semilobar HPE was the most common subtype of HPE, seen in $50 \%$ of the participants. Neurodevelopmental disabilities were found to correlate with HPE subtype. Factors associated with long-term survival included HPE subtype not alobar, female gender, and nontypical facial features. Four participants had de novo pathogenic variants in ZIC2. WES analysis of 11 participants did not reveal plausible candidate genes, suggesting complex inheritance in these cases. Indeed, in two probands there was a history of uncontrolled maternal type 1 diabetes.

Conclusion: Individuals with various HPE subtypes can survive into adulthood and the neurodevelopmental outcomes are variable. Based on the facial characteristics and molecular evaluations, we suggest that classic genetic causes of HPE may play a smaller role in this cohort.

Genet Med advance online publication 22 June 2017

Key Words: DISP1; holoprosencephaly; natural history; wholeexome sequencing; ZIC2

\section{INTRODUCTION}

Holoprosencephaly (HPE, MIM 236100) is defined by failed or incomplete division of the forebrain that occurs early in gestation. The etiology of HPE is highly heterogeneous and includes chromosomal anomalies, syndromic Mendelian disorders, environmental factors, and heterozygous variants in several HPE-associated genes. ${ }^{1-4}$ HPE occurs in up to 1 in 250 conceptions, ${ }^{5}$ and in approximately 1 in 10,000 live births. ${ }^{6,7}$ Nonsyndromic HPE (isolated brain and craniofacial anomalies) accounts for approximately $30-40 \%$ of all HPE cases seen at birth. ${ }^{8,9}$ Heterozygous mutations or small copynumber variations (CNVs) in SHH (MIM 600725), SIX3 (MIM 603714), ZIC2 (MIM 603073), and TGIF (MIM 602630) are found in approximately $30 \%$ of nonsyndromic cases, ${ }^{2,10}$ while mutations in any of over 10 additional genes have been seen at a much lower frequency. ${ }^{3,11}$ Familial cases of nonsyndromic HPE with dominant inheritance and variable expressivity or incomplete penetrance have been reported previously in multiple cohorts, ${ }^{12,13}$ pointing to the possibility of complex inheritance in these families. Recent case reports of digenic inheritance ${ }^{14,15}$ and studies on geneenvironment interactions in mice have supported this scenario of complex inheritance in HPE. ${ }^{16}$

Presenting as a spectrum of craniofacial and radiological findings, there are five HPE subtypes, classified by the degree of telencephalic fusion seen on brain imaging. These five subtypes, listed in decreasing order of severity, include alobar, semilobar, lobar, middle interhemispheric (MIH), and the septopreoptic types. ${ }^{17,18}$ The failed forebrain division in HPE is also associated with a spectrum of craniofacial malformations including synophthalmia, proboscis, closely spaced eyes, absent nasal bone, midline cleft lip and palate (with premaxillary agenesis or hypogenesis), and a single central maxillary incisor. The term "microform" is applied in the case

${ }^{1}$ Medical Genetics Branch, National Human Genome Research Institute, National Institutes of Health, Bethesda, Maryland, USA; ${ }^{2}$ Speech and Language Pathology Section, Department of Rehabilitation Medicine, Clinical Center, National Institutes of Health, Bethesda, Maryland, USA; ${ }^{3}$ National Institute of Neurological Disorders and Stroke, National Institutes of Health, Bethesda, Maryland, USA; ${ }^{4}$ Pediatrics and Developmental Neuroscience Branch, National Institute of Mental Health, Bethesda, Maryland, USA; ${ }^{5}$ Ophthalmic Genetics and Visual Function Branch, National Eye Institute, National Institutes of Health, Bethesda, Maryland, USA; ${ }^{6}$ Stanford University School of Medicine and Lucile Packard Children's Hospital, Stanford, California, USA; ${ }^{7}$ Neurology and Developmental Medicine, Kennedy Krieger Institute, Baltimore, Maryland, USA; ${ }^{8}$ Department of Neurology, Texas Scottish Rite Hospital for Children, Dallas, Texas, USA; ${ }^{9}$ Neurology and Neurotherapeutics, University of Texas Southwestern Medical Center at Dallas, Dallas, Texas, USA. Correspondence: Maximilian Muenke (mmuenke@nhgri.nih.gov) 
of an individual who presents with facial features typical for HPE but upon imaging is seen not to have brain abnormalities. In many cases there is a correlation between the severity of the craniofacial malformations and the severity of the HPE subtype seen on imaging, ${ }^{19}$ although there are exceptions. $^{20,21}$

Based on studies from the 1990s, first-year survival rates of newborns with nonsyndromic HPE are 30-50\%. 6,8,22 However, there is no published data on the long-term survival rates and natural history of these individuals. In this study, we evaluated a rare cohort of adolescents and adults with HPE with aims of characterizing genotypic and phenotypic features of the group, identifying factors associated with survival, and providing prognostic and natural history information so that information shared with families might be improved.

\section{Subjects}

\section{MATERIALS AND METHODS}

We evaluated 20 individuals with HPE, all at least 15 years old. Participants were secured through the International HPE Database and Registry from the Carter Centers for Brain Research in HPE and Related Brain Malformations (Carter Centers) at Texas Scottish Rite Hospital for Children (TSRHC) in Dallas, TX. We contacted families listed in the Carter Center registry who had children above the age of 15 years. Most of these individuals had been followed since birth or shortly after birth; some had undergone genetic testing in the past, while others had not. Previous results of genetic testing did not affect whether or not an individual was included in the study. The guardians of all subjects consented to our institutional review board-approved protocol (ClinicalTrials.gov: NCT00088426), which included permissions for the publication of photographs. Eleven participants and their families were evaluated at the Clinical Center of the National Institutes of Health (NIH) located in Bethesda, MD. The clinical evaluation entailed a detailed history and physical exam, neuropsychological testing, ophthalmic exam, abdominal ultrasound, modified barium swallow study, evaluation by rehabilitation medicine, and a brain magnetic resonance imaging (MRI) scan (or review of previous scans). For the nine subjects who were not able to visit the NIH, we reviewed medical records, obtained photographs, and collected blood samples from relevant family members.

\section{International HPE database and registry}

We compared our cohort's phenotypic data to those of 425 individuals from the International HPE Database and Registry. These individuals were enrolled prospectively from 1998 to 2015 through the Carter Centers. 279 subjects were clinically evaluated at one of the three Carter Centers (Kennedy Krieger Institute, Baltimore MD, TSRHC, Dallas TX, and Stanford University Medical Center, Stanford CA). Data were obtained for an additional 171 subjects whose parents remotely completed and submitted an online survey and either a computed tomography (CT) scan or an MRI scan without traveling to one of the three Carter Centers. The studies were approved by the institutional review board at each of the centers. The participants in this registry were referred through various sources, including pediatricians, neonatologists, geneticists, and neuroradiologists. Some were self-referred via the Carter Centers' website or "Families for HoPE," a support group for individuals with a family member with HPE. As part of the inclusion criteria, each of the participants had a CT or an MRI scan evaluated by a neuroimaging team at one of the Carter Centers to confirm the diagnosis of HPE and determine the HPE subtype.

\section{MRI scans}

Nineteen participants had brain MRI studies completed while one participant had only a CT scan available. All scans were read by the same radiologist at the Carter Centers. The subtype of HPE was determined based on the extent of the interhemispheric separation and ventricular morphology. The degrees of nonseparation of the deep gray nuclei, including the caudate nuclei, lentiform nuclei, thalamic nuclei, and hypothalamic nuclei, were graded using previously published methods. ${ }^{23}$ Briefly, the neuroimaging studies were graded according to the degree of separation on a scale of 0 (fully separated) to 3 (complete noncleavage) for all deep gray structures except for the hypothalamic nuclei, where a 0 -to-2 scale was used. Spatial orientation was also evaluated, as was the relationship between the basal ganglia and the diencephalic structures and mesencephalon. The interpretation was blinded to the clinical findings of the subjects.

\section{Genetic testing}

Genetic testing was performed by chromosomal microarray, Sanger sequencing of the four most common HPE-associated genes (SHH, ZIC2, SIX3, and TGIF), and whole-exome sequencing (WES). All of our participants provided informed consent for genetic testing in accordance with the guidelines of our institutional review board-approved protocol (ClinicalTrials.gov: NCT00645645). Approximately half of the participants had four-gene testing prior to their participation in the study and only a few had chromosomal microarray testing. We repeated four-gene testing for individuals who underwent testing outside of our lab. WES was performed when samples for trios (proband, mother, and father) were available. Briefly, Sanger sequencing was performed using standard methods. Chromosomal microarray was performed using the HumanOmniExpressExome BeadChip (version 1.2-B, Illumina, San Diego, CA, USA), following the Illumina Infinium assay protocol. The samples were scanned by the Illumina iScan system, and clustering and genotype calling were performed using the GenomeStudio Genotyping Module (version 2011.1, Illumina). CNV detection was performed using cnvPartition (version 3.2, Illumina), and Nexus Copy Number (version 8.0, BioDiscovery, El Segundo, CA, USA). WES was performed using the SeqCap EZ Exome + UTR version 3.0 capture kit (Roche NimbleGen, Madison, WI, USA) and the Illumina HiSeq2500 platform. Sequencing data were aligned to the human 
reference genome using Novoalign V3.02.07 (Novocraft Technologies, Selangor, Malaysia). Variants were called using the in-house MPG genotype caller. Average coverage attained was $65 \mathrm{x}$ with on average $90 \%$ of targeted bases covered at 10x. Variants that survived filtering are shown in the Supplementary Note online. We included exonic variants (nonsense, frameshift and splicing, missense, and in-frame indels) that fit a de novo dominant or recessive mode of inheritance with a minor allele frequency of $<0.005$ in the Exome Aggregation Consortium database (ExAC). Because of the possibility of incomplete penetrance, we investigated all exonic variants in genes previously reported in HPE (gene list in Supplementary Note) with a minor allele frequency of $<0.0001$ in ExAC.

\section{Neuropsychological evaluation}

In conditions with widely varying cognitive functioning, numerous studies ${ }^{24}$ have recommended utilizing either fullscale intelligence quotient (IQ) or ratio IQ based on mental age/chronological age, if standardized scores are not available. As such and based on initial observation of the individual and parent report, we administered one of the following tests: Wechsler Intelligence Scale for Children - Fourth Edition, ${ }^{25}$ Wechsler Abbreviated Scale of Intelligence - Second Edition, ${ }^{26}$ Mullen Scales of Early Learning, ${ }^{27}$ and the Vineland Adaptive Behavior Scales, Second Edition. ${ }^{28}$ This hierarchical test administration procedure is commonly used in studies of neurological disorders.

\section{Ophthalmic exam}

A complete ophthalmic exam was performed on participants who visited the NIH Clinical Center. This included assessment of visual function, external exam and motility, anterior segment exam using a slit lamp (a portable slit lamp for participants with reduced cooperation), dilated fundus exam, and cycloplegic refraction. Optical coherence tomography scans, fundus photography, and electroretinography were obtained when indicated and when allowed by the participants' level of cooperation.

\section{Swallow assessment}

Swallowing function was assessed through administration of the NIH Modified Barium Swallow instrumental assessment, which consists of discrete swallows of $5-10 \mathrm{ml}$ for liquids, $5 \mathrm{ml}$ for puree, and $1 / 4$ graham cracker, if the patient is able. The American Speech Hearing Association (ASHA) swallowing outcome and diet restriction scales ${ }^{29}$ were applied to each subject's swallowing function by the speech language pathologist.

\section{RESULTS}

Our study cohort included 20 subjects from 19 families, consisting of 14 females and 6 males (2.3:1 female-to-male ratio). The age range was 15 to 33 years, with a mean of 22.5 years. Except for one family with two affected siblings, all cases were simplex, with no known family history of HPE or associated craniofacial anomalies such as midline cleft lip/ palate or a single central maxillary incisor. In two cases, prenatal history was significant for uncontrolled maternal type 1 diabetes with ketoacidosis during early gestation. For 10 subjects $(50 \%)$ the diagnosis was delayed, ranging between the ages of 2 months and 17 years. The HPE subtypes observed in the cohort included: semilobar in 10 subjects (50\%), lobar in three subjects (15\%), MIH in five subjects (25\%), and septopreoptic in one subject. For one subject a CT scan was the only available assessment for subtype classification and was interpreted as showing alobar HPE. Using the Carter Centers' International HPE Database and Registry as a reference guide, the distribution of HPE subtypes was similar to that found in the pediatric population except in the case of alobar HPE, which was less frequent in this cohort $(P=$ $<0.0001$, Fisher's exact test) (Figure 1).

Variable degrees of neurodevelopmental disability were found in the cohort. The majority of subjects $(60 \%)$ were on the severe end of the spectrum. They were nonambulatory with minimal hand function and fully dependent on caregivers. These individuals were nonverbal but frequently communicated with their families by means of eye gaze devices and facial gestures. They were mostly alert and able to understand social situations as well as to recognize family members. Four subjects (20\%) were intermediate, with milder motor impairments. They were able to manipulate a wheelchair, use a tablet, stand, or walk a few steps. These individuals communicated verbally or with an electronic communication device at various levels. Four subjects (20\%) were on the relatively mild end of the spectrum, with no or minimal motor dysfunction, and communicated verbally at a preschool level or above. Of note, there was a correlation between the HPE type seen on imaging and the degree of neurodevelopmental disability observed. (Table 1, Figure 1).

Eleven participants each had at least some component of a neuropsychological evaluation done. Individuals with semilobar HPE received the lowest scores on the Vineland and full IQ assessments. For individuals with milder HPE types, there was a variety of scores, ranging from mild to severe intellectual delay (ID). Areas of relative strength were reading, communication, socialization, and daily living. Lower scores were received for auditory and reading comprehension, math, visual discrimination, and working memory skills. Psychiatric disorders such as obsessive compulsive disorder, depression, anxiety, psychosis, and attention deficit disorder were reported in $4 / 9$ adults in the intermediate or mild category. Treatment with antidepressant or anti-anxiety medications was beneficial in most cases.

Twelve subjects $(60 \%)$ had a gastrostomy tube (G-tube), while nine (45\%) were strictly G-tube-fed, owing to a history of aspiration. Seven of the 11 individuals who were fed by mouth had dietary modifications to facilitate feeding. There was a positive correlation between strict G-tube feeding and having a more severe HPE subtype as seen on imaging (Figure 1). Interestingly, swallowing characteristics were similar in 6/7 subjects with different HPE subtypes 
a
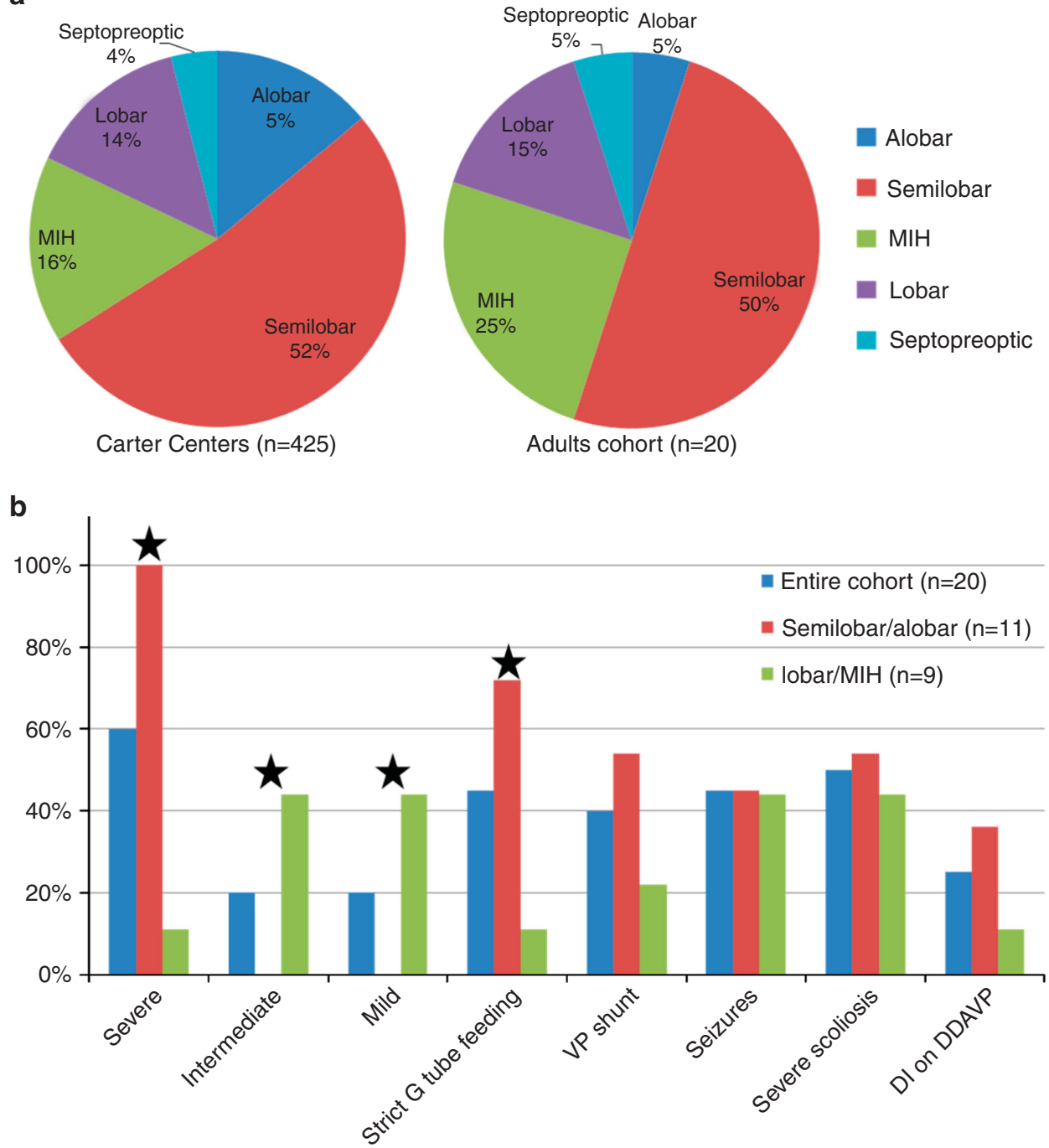

Figure 1 Distribution of HPE subtypes and prevalence of common clinical findings. (a) The distribution of HPE subtypes in the this cohort compared to the International HPE Database and Registry. The distribution of HPE types was similar in the two groups, except for alobar HPE, which was less frequent in this cohort (Fischer's test, $P=<0.0001$ ). (b) Clinical findings in semilobar and alobar HPE versus milder types. Severe neurodevelopmental disabilities and strict G-tube feeding were more common in individuals with semilobar or alobar HPE than in individuals with milder HPE types (Fischer's test $P=0.0001$ and 0.009 respectively). Severe =nonambulatory, limited hand function, and nonverbal. Intermediate $=$ intermediate motor disabilities with basic verbal communication. Mild = minor motor disabilities and verbal communication at a preschool level or above. DDAVP, desmopressin; DI, diabetes insipidus; HPE, holoprosencephaly; MIH, middle interhemispheric; VP, ventriculoperitoneal. Stars indicate that the difference between the two groups is statistically significant.

(Table 1). They had diminished bolus formation, reduced volitional bolus transfer, nutritive suckles, and delayed pharyngeal swallow responses without aspirations that were equivalent to outcome level 6 (swallow is safe and the individual eats with rare cueing provided). However, when we used the ASHA diet restrictions scale to assess swallowing abilities based on the diet history in the cohort, we saw a variety of outcomes, ranging from level 1 to level 6 (Table 1).

A summary of the clinical history is shown in Table 2. Nine subjects $(45 \%)$ had seizures, which were controlled with one or two medications in the majority of cases. Two (10\%) had refractory seizures and overall a more severe phenotype. Eight subjects (40\%) had congenital hydrocephalus requiring a ventriculoperitoneal shunt and four (20\%) also had a history of a parietal encephalocele. Five subjects (25\%) had diabetes insipidus (DI) and an additional three had subclinical DI with borderline hypernatremia controlled with fluids versus medication. Other pituitary abnormalities were uncommon. Several subjects had primary hypothyroidism without pituitary deficiencies. All subjects had normal sexual development and LH, FSH levels, except for one subject with a history of precocious puberty.

The majority of subjects had various ophthalmic abnormalities. The most common were cortical visual impairment, hypo-/hypertelorism, ptosis/lagophthalmos, dry eyes, strabismus, and optic nerve abnormalities (including optic atrophy 
Table 1 The spectrum of neurodevelopmental disabilities in adolescents and adults with HPE

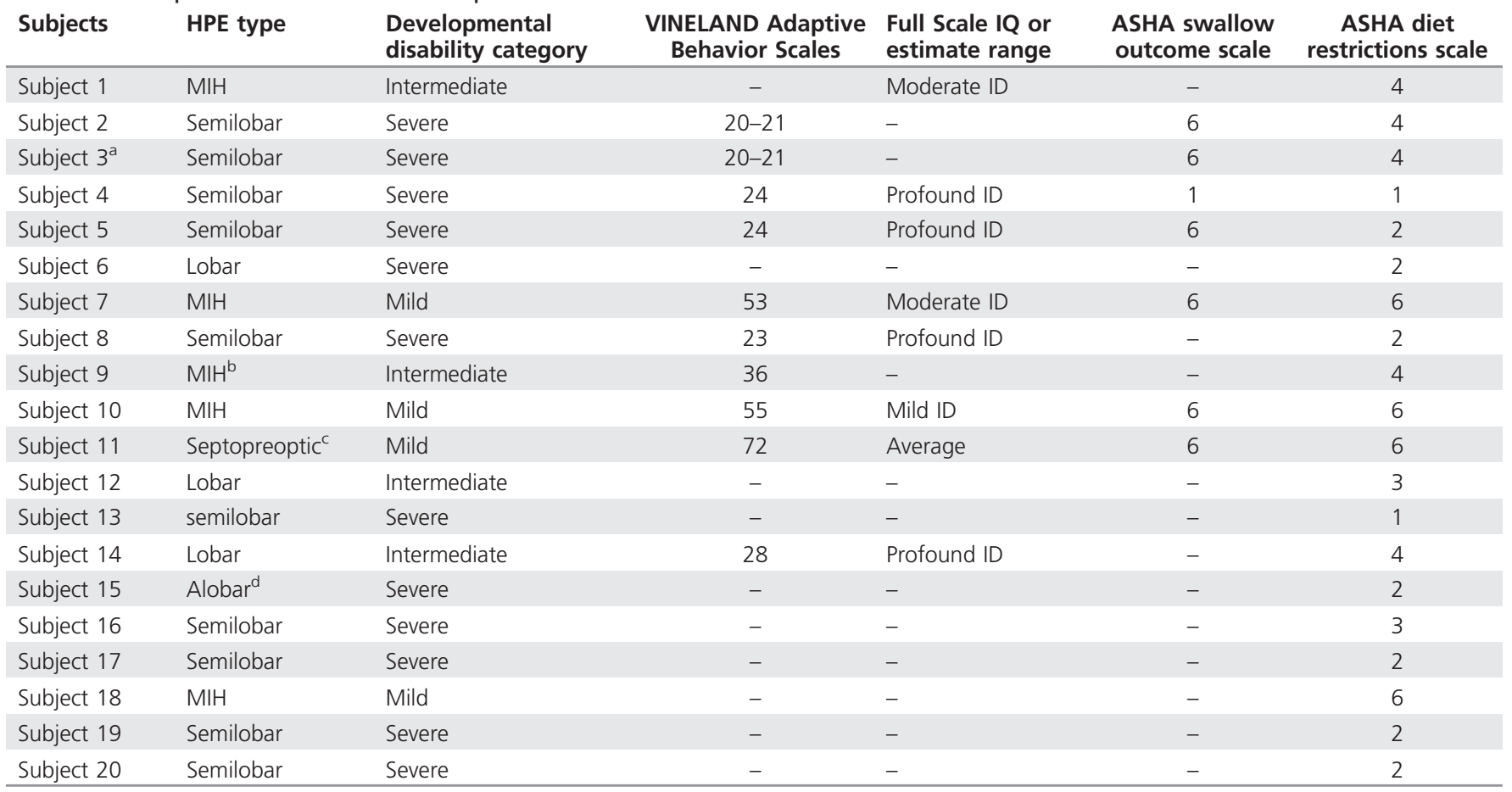

ASHA, American Speech Language Hearing Association; HPE, holoprosencephaly; ID, intellectual disability; IQ, intelligence quotient; MIH, middle interhemispheric.

The WASI-II was administered to subject 10; the Mullen was administered to subjects 4, 5, 8, and 14; the WAIS-IV was administered to subjects 7 and 10 . The WISC-IV was administered to subject 1 , who was not able to complete the test. His score is based on the general ability index. Full Scale IQ or estimate ranges: average =average range (85-115); borderline =71-84; mild ID =55-70; moderate ID =40-55; severe ID =26-39; profound ID = $<25$.

ASHA diet restriction scale: $1=$ individual unable to swallow anything safely by mouth; 2 =individual not able to swallow safely by mouth for nutrition and hydration; $3=$ individual taking less than $50 \%$ of nutrition and hydration by mouth; $4=$ swallowing is safe but moderate cues are required to use compensatory strategies, or the individual has moderate diet restrictions, or requires some gastrostomy-tube feeding; $5=$ swallowing is safe with minimal diet restriction and cueing, all nutritional needs are met per mouth; 6 =swallowing is safe and the individual eats and drinks independently and requires cueing rarely. They may need to avoid specific foods or require additional time; $7=$ Swallowing is safe and efficient.

a Sibling of subject 2; ${ }^{b}$ with dysplastic cortex; ' patient was previously described by Hahn et al.; ${ }^{17}$ dbased on a computed tomography scan.

and hypoplasia). Microcornea was present in three subjects examined at the NIH Clinical Center. None of the subjects examined at NIH showed evidence of ocular coloboma. One subject was noted to have a pigmentary retinopathy, a finding that has not been previously associated with HPE. Supplementary Table S1 summarizes the ophthalmic exam findings of HPE subjects examined at NIH.

Only five subjects (25\%) had typical facial features associated with HPE; the rest had subtle and nonspecific dysmorphic features. Some participants had subtle facial characteristics that are similar to those typically found in subjects with ZIC2 mutations ${ }^{20}$ (Figure 2), even though they did not have ZIC2 variants. The majority of the cohort had microcephaly, although three individuals with no history of hydrocephalus had a normal head circumference. Short stature was common, with a mean final height of $150 \mathrm{~cm}$ in males and $146 \mathrm{~cm}$ in females (Table 2).

Sanger sequencing of SHH, ZIC2, SIX3, and TGIF was performed on all subjects. Four subjects had a positive result showing a truncating mutation in ZIC2 (Table 3). Chromosomal microarray was performed in 17/20 subjects and was negative for $\mathrm{CNVs}$ in known HPE-related loci. One participant with septopreoptic HPE had a complex CNV on chromosome 8, including a deletion of FGFR1 (Table 3). The contribution of this CNV to HPE is unclear. WES was performed for 11 subjects from 10 families who had a negative four-gene testing and for whom samples were available for a trio analysis. Recessive and dominant variants that survived filtering are shown in the Supplementary Note. We detected a total of six high-quality de novo exonic variants in four subjects. The variants were in genes not known to be involved in brain development and not seen in a larger cohort of individuals with HPE (unpublished data). Investigation of dominantly inherited variants in genes previously associated with HPE revealed a variant of uncertain significance (VUS) in DISP1 (MIM 607502).

\section{Survival}

\section{DISCUSSION}

There is a common misperception that the vast majority of children with HPE have very limited survival rates. Previous studies support low survival rates in newborns with isolated nonsyndromic alobar HPE, ${ }^{9,22}$ but there have not been accurate data on survival of individuals with other HPE subtypes. In this study, we show that $50 \%$ of adolescents and adults with HPE have semilobar HPE. This frequency is 

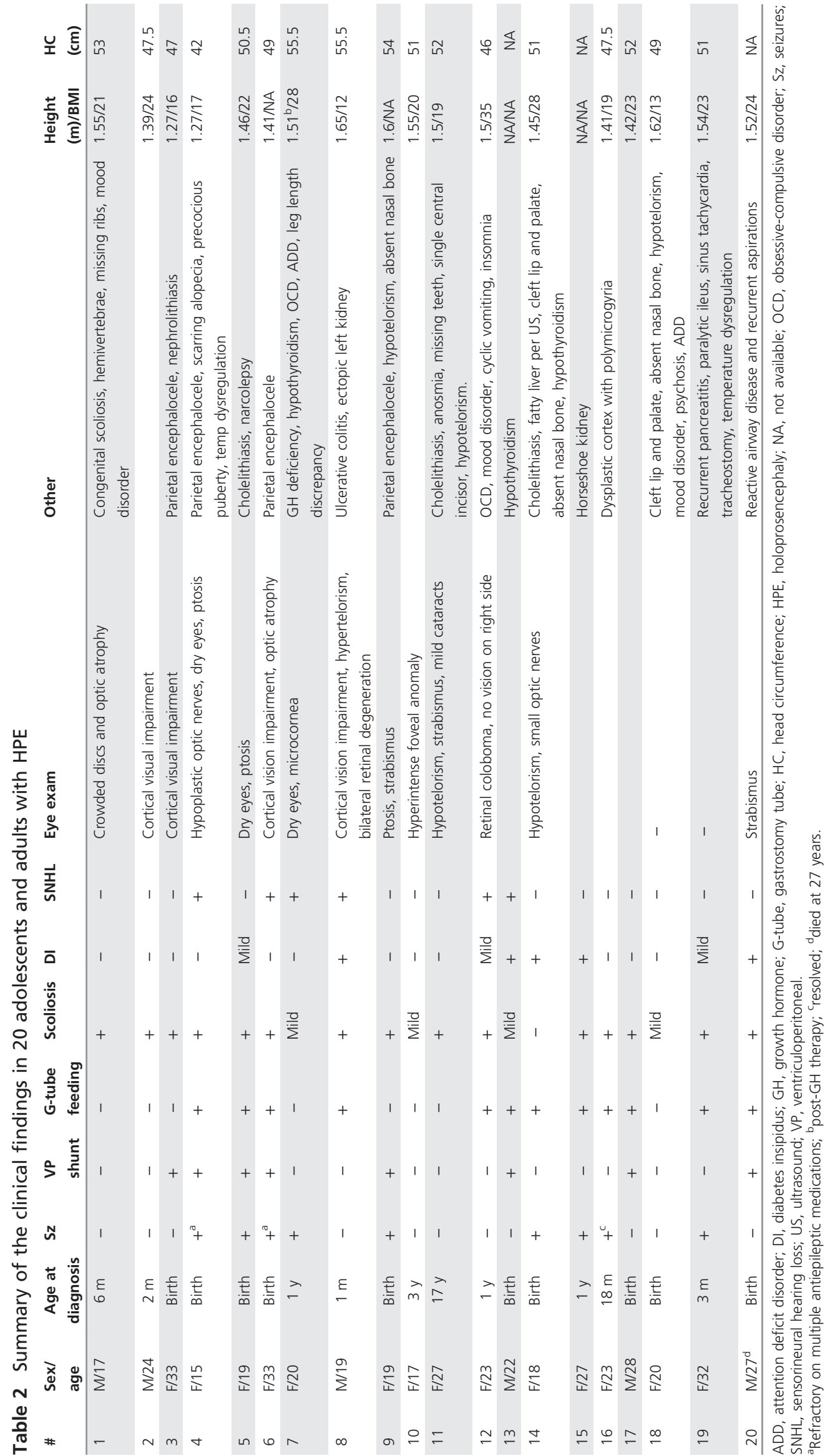




Figure 2 Facial photographs of 12 adolescents and adults with holoprosencephaly (HPE). Four out of 20 individuals had typical HPE features. d had closely spaced eyes and a single maxillary central incisor (picture after repair). e had closely spaced eyes, absent nasal bone, midface hypoplasia and cleft lip and palate. $f$ had closely spaced eyes, missing nasal bone, and midface hypoplasia. I had midface hypoplasia and cleft lip and palate. Several subjects had features similar to those previously described in individuals with ZICZ mutations, although their ZIC2 test results were negative. These features include: bitemporal narrowing (c, g, k), upslanting palpebral fissures (b, $c, g, j, k, n)$, a short nose with upturned nares (b, $c$, k), a broad and/or deep philtrum (b, $c, j)$ and large ears (b, c, g, j, k). Consent to publish the photos was obtained for all subjects.

similar to that observed in the Carter Centers' registry of 425 subjects, indicating that prolonged survival is potentially frequent in individuals with this relatively severe subtype. Longer survival may be attributed to the improvement in neonatal and pediatric medical care over time, along with advances in imaging studies which allow for detection of milder cases of HPE with increased life spans.

\section{Clinical findings}

Our study participants had several characteristics that differed from those reported in other HPE studies. The vast majority of the cases in this study were simplex, with a negative family history for HPE spectrum. Our cohort had a higher female-tomale ratio than previously reported (2.3 vs. 1.2). ${ }^{9,15}$ Most subjects had only mild facial dysmorphism with none of the 
Table 3 Subjects with positive genetic testing

\begin{tabular}{|c|c|c|c|c|c|}
\hline Subject no. & Gene & Inheritance & Variant & Transcript & Interpretation \\
\hline 13 & ZICZ2 & De novo & c.1095_1096del, p.Cys365* & NM_007129 & Pathogenic \\
\hline 16 & ZICZ & De novo & c. $1148 \_1464 d e l$, p.Ser482Argfs*42 & NM_007129 & Pathogenic \\
\hline 20 & ZIC2 & De novo & c. 793 C > T, p.G $\ln 265^{*}$ & NM_007129 & Pathogenic \\
\hline 15 & ZICZ2 & Unknown & c.1097_1098del, p.Glu366Valfs*2 & NM_007129 & Pathogenic \\
\hline 14 & DISP1 & Maternally inherited & c.743C > T:p.Ala248Val & NM_032890 & Variant of uncertain significance \\
\hline 11 & CNV & Unknown & $\begin{array}{l}\text { arr[hg19] 8p21.2-p.21.1(27141452-28 } \\
8 p 12-p 11.21(35754100-39710324) \times 1 \\
8 p 11.22-p 11.21(39720982-40167696)\end{array}$ & $\begin{array}{l}479611) \times 1 \\
x 3\end{array}$ & $\begin{array}{l}\text { Pathogenic but association with } \\
\text { holoprosencephaly is unclear }\end{array}$ \\
\hline
\end{tabular}

CNV, copy-number variant.

classic HPE features. Specifically, none of our participants with semilobar HPE had typical facial features. The lack of classic facial features in this cohort also explains the reported delay in diagnosis beyond 2 months of age in more than 50\% of the subjects. Mercier et al. reported mild typical HPE features or nonspecific dysmorphism in $40 \%$ of 645 probands with various HPE subtypes. ${ }^{21}$ An association between less severe facial features and survival was previously reported in studies on newborns. ${ }^{8,9}$ In our study, which is the first study to show a correlation between facial features and survival in adults, $17 / 20$ had mild or nonspecific features, supporting a correlation with long-term survival $(P=0.0001$, Fisher's exact test).

Another association found in this study is one between the degree of neurodevelopmental disability and HPE subtype, where more severe disability was associated with the more severe semilobar subtype. Interestingly, the frequency of seizures was the same in both the severe and the milder group $(\sim 40 \%)$ (Figure 1), indicating that seizure risk may depend on factors other than the degree of forebrain fusion, such as cortical dysplasia. Of note, seizures in most individuals in this cohort were well controlled with medication, except in the two individuals with refractory seizures. Compared to previous reports, ${ }^{30,31}$ DI was less prevalent in this study ( $25 \%$ vs. $54-70 \%)$. This may be the result of a bias toward milder HPE subtypes, since there is a correlation between the degree of hypothalamic separation and the presence of DI. ${ }^{32,33}$ Speech and swallow evaluations performed on seven individuals showed that some subjects had the ability to swallow modified textures safely. Still, many individuals with HPE require a $G$ tube to manage diabetes insipidus, receive enough nutrition, and prevent occasional aspirations which may lead to severe complications.

Conventional neuropsychological testing can be particularly challenging in persons with profound disabilities, involving multiple systems; i.e., intellectual, motor, and others. ${ }^{34} \mathrm{We}$ implemented the Vineland Adaptive Behavior Scales to assess five participants with semilobar HPE. Because the Vineland scale includes four domains, with only one for socialization, the overall adaptive behavior composite scores were generally very low. These results support further development of specialized tests such as the Carter Neurocognitive. ${ }^{35}$ Nevertheless, in this study, we used an alternative test that includes existing standardization (Mullen Scales of Early Learning) when possible. For subjects with milder HPE types, their Vineland scores correlated well with their scores from directly administered measures of neurocognitive functioning.

There are limited reports in the literature about psychiatric abnormalities in individuals with HPE. ${ }^{36}$ In our cohort, we found that 4/9 subjects with milder HPE subtypes were treated for mood disorders or other psychiatric conditions. In all cases, treatment had reportedly led to clinical improvement. We speculate that a range of psychiatric diagnoses may be prevalent among adolescents and adults with milder HPE types, as seen in this cohort. Therefore, an evaluation by a psychologist or psychiatrist is important especially in older children and adolescents.

A number of ophthalmic findings have been reported in HPE, including cyclopia, hypotelorism, uveal coloboma, and optic nerve hypoplasia. Microform HPE ( $\mathrm{SHH}$-related) has also been found to present with ocular pathology. ${ }^{37}$ In this cohort, we also identified a number of previously reported ocular abnormalities associated with HPE (hypotelorism, ptosis, strabismus, microcornea, optic nerve hypoplasia). ${ }^{38}$ Furthermore, we found some previously unreported ophthalmic findings, namely dry eyes/corneal scarring and a retinal degeneration in one participant. These manifestations may be more difficult to detect in younger subjects and may worsen with age; it is therefore important to raise awareness regarding these ocular problems in older HPE subjects. Therapy for dry eyes is readily available and should be considered after an assessment by the ophthalmologist for individuals who show signs and symptoms of the condition (red eyes, tearing, eye rubbing).

\section{Etiology}

Several factors point to an different etiology in adolescents and adults with HPE from that in newborns and young children with the disorder. We tested the four genes ( $\mathrm{SHH}$, SIX3, ZIC2, TGIF) most commonly associated with HPE using Sanger sequencing and chromosomal microarray to find truncating mutations in ZIC2 in 4 subjects (20\%) who had semilobar or alobar HPE. Interestingly, there were no subjects with SIX3 or SHH variants, which typically occur as frequently as ZIC2 variants in general HPE cohorts. ${ }^{2}$ This may indicate that individuals with ZIC2 mutations are more 
likely to survive longer, even in the presence of severe HPE subtypes. In turn, this raises the question of whether the increased survival is related to the specific developmental stage disrupted by ZIC2 mutations in early embryogenesis. As opposed to other HPE genes in the SHH pathway, ZIC2 acts in mid-gastrulation and disrupts nodal signaling. ${ }^{39}$ This may also may help explain why individuals with ZIC2 mutations are less likely to have classic HPE facial features, ${ }^{20}$ as seen in individuals in this cohort.

Chromosomal microarray did not identify CNVs in known HPE-related loci. However, one participant had a large CNV on 8p that included FGFR1 (MIM 136350) and 47 other protein coding genes. Haploinsufficency of FGFR1 is associated with hypogonadotropic hypogonadism, and certain missense variants in FGFR1 are associated with Hartsfield syndrome (MIM 615465). ${ }^{14}$ WES analysis was negative for FGFR1 variants in this individual, who had history of anosmia with normal sexual development and FSH/LH levels at 27 years. We are not aware of additional reports of HPE in this locus. Additional genes within the CNV with a PLi above 0.9 were PTK2B, ASH2L, LSM1, and WHSC1L1.

Analysis of the exome data in 10 families did not reveal pathogenic variants. One subject had a VUS in DISP1 inherited from an unaffected mother (Table 3). This substitution is not seen in ExAC and affects a highly conserved residue. It was recently suggested that variants in this gene may contribute to recessive or digenic inheritance in HPE. ${ }^{11}$ Although we considered DISP1 a candidate gene, based on its function and presence in the HPE10 locus, ${ }^{40}$ we have not yet found sufficient evidence to suggest causation. Since the majority of cases in this cohort were simplex with a negative family history, we expected to identify de novo variants in candidate genes. But that was not the case, as investigation of de novo exonic variants using WES revealed no conclusive candidates in 11 subjects. Instead, our results support the presence of a more complex inheritance in the group, further highlighting the significant role that environmental factors may play in HPE (as in the two presented cases with a history of uncontrolled type 1 diabetes mellitus in early gestation).

In conclusion, results from our study evaluating a rare cohort of adolescents and adults with HPE suggest that individuals with various HPE subtypes can survive into adulthood. The neurodevelopmental disabilities that can occur in this group are variable and are shown on imaging to correlate with the HPE subtype. Factors associated with long-term survival include HPE subtypes other than alobar, female gender, nontypical facial features, and potentially, ZIC2 mutations. As individuals with HPE get older, special concern should be given to spasticity-related complications, and psychiatric and ophthalmic abnormalities that may not be present during childhood.

\section{SUPPLEMENTARY MATERIAL}

Supplementary material is linked to the online version of the paper at http://www.nature.com/gim

\section{ACKNOWLEDGMENTS}

We thank the participants and their families. This work was supported by the intramural program of the National Human Genome Research Institute, NIH. The NIH Intramural Sequencing Center (NISC) conducted the sequencing experiments in this project.

\section{DISCLOSURE}

The authors declare no conflict of interest.

\section{REFERENCES}

1. Muenke M, Beachy PA. Genetics of ventral forebrain development and holoprosencephaly. Curr Opin Genet Dev 2000;10:262-269.

2. Roessler E, Velez Jl, Zhou N, Muenke M. Utilizing prospective sequence analysis of $\mathrm{SHH}, \mathrm{ZIC2}, \mathrm{SIX} 3$ and TGIF in holoprosencephaly probands to describe the parameters limiting the observed frequency of mutant genexgene interactions. Mol Genet Metab 2012;105:658-664.

3. Solomon BD, Gropman A, Muenke M. Holoprosencephaly overview. In: Pagon RA, Adam MP, Ardinger HH, et al. (eds). GeneReviews. University of Washington: Seattle, WA, 1993.

4. Gripp KW, Wotton D, Edwards MC, et al. Mutations in TGIF cause holoprosencephaly and link NODAL signalling to human neural axis determination. Nat Genet 2000;25:205-208.

5. Matsunaga E, Shiota K. Holoprosencephaly in human embryos: epidemiologic studies of 150 cases. Teratology 1977;16:261-272.

6. Bullen PJ, Rankin JM, Robson SC. Investigation of the epidemiology and prenatal diagnosis of holoprosencephaly in the North of England. Am J Obstet Gynecol 2001;184:1256-1262.

7. Leoncini E, Baranello G, Orioli IM, et al. Frequency of holoprosencephaly in the international clearinghouse birth defects surveillance systems: searching for population variations. Birth Defects Res A Clin Mol Teratol 2008;82:585-591.

8. Olsen CL, Hughes JP, Youngblood LG, Sharpe-Stimac M. Epidemiology of holoprosencephaly and phenotypic characteristics of affected children: New York state, 1984-1989. Am J Med Genet 1997;73:217-226.

9. Croen LA, Shaw GM, Lammer EJ. Holoprosencephaly: epidemiologic and clinical characteristics of a California population. Am J Med Genet 1996;64:465-472.

10. Bendavid C, Rochard L, Dubourg C, et al. Array-CGH analysis indicates a high prevalence of genomic rearrangements in holoprosencephaly: an updated map of candidate loci. Hum Mutat 2009;30:1175-1182.

11. Dubourg C, Carre W, Hamdi-Roze $\mathrm{H}$, et al. Mutational spectrum in holoprosencephaly shows that Fgf is a new major signaling pathway. Hum Mutat 2016; 37:1329-1339.

12. Solomon BD, Bear KA, Wyllie A, et al. Genotypic and phenotypic analysis of 396 individuals with mutations in Sonic Hedgehog. J Med Gen 2012;49:473-479.

13. Lacbawan F, Solomon BD, Roessler E, et al. Clinical spectrum of SIX3associated mutations in holoprosencephaly: correlation between genotype, phenotype and function. J Med Genet 2009;46:389-398.

14. Hong S, Hu P, Marino J, et al. Dominant-negative kinase domain mutations in FGFR1 can explain the clinical severity of Hartsfield syndrome. Hum Mol Genet 2016;25:1912-1922.

15. Mouden C, Dubourg C, Carre W, et al. Complex mode of inheritance in holoprosencephaly revealed by whole exome sequencing. Clin Genet 2016;89:659-668.

16. Kietzman HW, Everson JL, Sulik KK, Lipinski RJ. The teratogenic effects of prenatal ethanol exposure are exacerbated by Sonic Hedgehog or GLI2 haploinsufficiency in the mouse. PloS One 2014;9:e89448.

17. Hahn JS, Barnes PD. Neuroimaging advances in holoprosencephaly: refining the spectrum of the midline malformation. Am J Med Genet $C$ Semin Med Genet 2010;154C:120-132.

18. Barkovich AJ, Simon EM, Clegg NJ, Kinsman SL, Hahn JS. Analysis of the cerebral cortex in holoprosencephaly with attention to the sylvian fissures.Am J Neuroradiol 2002;23:143-150.

19. Demyer W, Zeman W, Palmer CG. The face predicts the brain: diagnostic significance of median facial anomalies for holoprosencephaly (arhinencephaly). Pediatrics 1964;34:256-263.

20. Solomon BD, Lacbawan F, Mercier S, et al. Mutations in ZIC2 in human holoprosencephaly: description of a novel ZIC2 specific phenotype and 
comprehensive analysis of 157 individuals. J Med Genet 2010;47: 513-524.

21. Mercier S, Dubourg C, Garcelon N, et al. New findings for phenotypegenotype correlations in a large European series of holoprosencephaly cases. J Med Genet 2011;48:752-760.

22. Barr M Jr, Cohen MM Jr. Holoprosencephaly survival and performance. Am J Med Genet 1999;89:116-120.

23. Simon EM, Hevner R, Pinter JD, et al. Assessment of the deep gray nuclei in holoprosencephaly. Am J Neuroradiol 2000;21:1955-1961.

24. Dickson PI, Pariser AR, Groft SC, et al. Research challenges in central nervous system manifestations of inborn errors of metabolism. Mol Genet Metab 2011;102:326-338.

25. Wechsler D. Wechsler Intelligence Scale for Children, Fourth Edition. San Antonio, TX: Psychological Corporation, 2003.

26. Wechsler D. Wechsler Abbreviated Scale of Intelligence, Second Edition (WASI-II). San Antonio, TX: NCS Pearson, 2011.

27. Mullen EM (ed). Mullen Scales of Early Learning. Circle Pines, MN: American Guidance Service, 1995.

28. Sparrow SS, Cicchetti DV, Balla DA. Vineland Adaptive Behavior Scales, Second Edition. Circle Pines, MN: AGS Publishing, 2005.

29. Outcome Measures Report Health Care Services Team American SpeechLanguage-Hearing Association, Rockville, MD healthservices@asha.org. 2003.

30. Hahn JS, Hahn SM, Kammann $\mathrm{H}$, et al. Endocrine disorders associated with holoprosencephaly. J Pediatr Endocrinol Metab 2005;18: 935-941.

31. Traggiai C, Stanhope R. Endocrinopathies associated with midline cerebral and cranial malformations. J Pediatr 2002;140:252-255.

32. Plawner LL, Delgado MR, Miller VS, et al. Neuroanatomy of holoprosencephaly as predictor of function: beyond the face predicting the brain. Neurology 2002;59:1058-1066.

33. Lewis AJ, Simon EM, Barkovich AJ, et al. Middle interhemispheric variant of holoprosencephaly: a distinct cliniconeuroradiologic subtype. Neurology 2002;59:1860-1865.

34. Vlaskamp $\mathrm{Na}$. A need for a taxonomy for profound intellectual and multiple disabilities. J Policy Pract Intellect Disabil 2007;4:83-87.
35. Leevers HJ, Roesler CP, Flax J, Benasich AA. The carter neurocognitive assessment for children with severely compromised expressive language and motor skills. J Child Psychol Psychiatry 2005;46:287-303.

36. Virta M, Launes J, Valanne L, Hokkanen L. Adult with middle interhemispheric variant of holoprosencephaly: neuropsychological, clinical, and radiological findings. ArchClin Neuropsychol 2016;31:472-479.

37. Bakrania P, Ugur Iseri SA, Wyatt AW, et al. Sonic hedgehog mutations are an uncommon cause of developmental eye anomalies. Am J Med Genet A 2010;152A:1310-1313.

38. Pineda-Alvarez $D E$, Solomon $B D$, Roessler $E$, et al. A broad range of ophthalmologic anomalies is part of the holoprosencephaly spectrum. Am J Med Genet A 2011;155A:2713-2720.

39. Houtmeyers R, Tchouate Gainkam O, Glanville-Jones HA, et al. Zic2 mutation causes Holoprosencephaly via disruption of NODAL signalling. Hum Mol Genet 2016;25:3946-3959.

40. Roessler E, Ma Y, Ouspenskaia MV, et al. Truncating loss-of-function mutations of DISP1 contribute to holoprosencephaly-like microform features in humans. Hum Genet 2009;125:393-400.

(c) (i) $(-)$ This work is licensed under a Creative Commons Attribution-NonCommercial-NoDerivs 4.0

International License. The images or other third party material in this article are included in the article's Creative Commons license, unless indicated otherwise in the credit line; if the material is not included under the Creative Commons license, users will need to obtain permission from the license holder to reproduce the material. To view a copy of this license, visit http://creativecommons.org/licenses/ by-nc-nd/4.0/

(C) The Author(s) 2018 